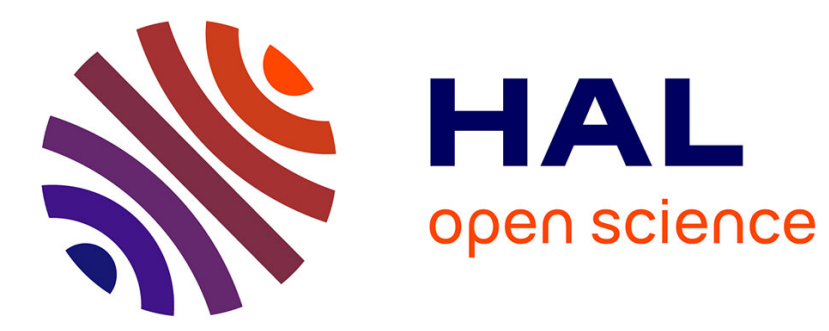

\title{
Eigensolutions of perturbed nearly defective matrices
}

Angelo Luongo

\section{To cite this version:}

Angelo Luongo. Eigensolutions of perturbed nearly defective matrices. Journal of Sound and Vibration, 1995, 185 (3), pp.377-395. hal-00801833

\section{HAL Id: hal-00801833 \\ https://hal.science/hal-00801833}

Submitted on 18 Mar 2013

HAL is a multi-disciplinary open access archive for the deposit and dissemination of scientific research documents, whether they are published or not. The documents may come from teaching and research institutions in France or abroad, or from public or private research centers.
L'archive ouverte pluridisciplinaire HAL, est destinée au dépôt et à la diffusion de documents scientifiques de niveau recherche, publiés ou non, émanant des établissements d'enseignement et de recherche français ou étrangers, des laboratoires publics ou privés. 


\title{
EIGENSOLUTIONS OF PERTURBED NEARLY DEFECTIVE MATRICES
}

\author{
A. LUONGO \\ Department of Structural Engineering, University of L'Aquila, Monteluco di Roio, \\ 67040 L'Aquila, Italy
}

\begin{abstract}
Perturbation methods for approximating eigenvalues and eigenvectors of perturbed nearly defective (mistuned) systems have been discussed. It is shown that the mistuning causes Taylor expansions to be not uniformly valid even in small intervals of the perturbation parameter, subsequently rendering them useless for practical purposes. The problem is overcome by starting the perturbation expansion from an exactly defective (tuned) system "close" to the mistuned one. Asymptotic expansions are then obtained in terms of fractional powers of two parameters: the modification and the mistuning parameter. However, as the tuned system is unknown, an inverse problem has to be solved in order to determine it. An algorithm valid for the particular but frequent case of several couples of nearly coincident eigenvalues has been detailed for this problem; an outline of a more general case is also given. Illustrative examples are presented.
\end{abstract}

\section{INTRODUCTION}

Perturbation methods are efficient tools for computing eigensolutions of perturbed matrices. They permit the construction of asymptotic expansions approximating the exact eigensolutions near known unperturbed values, when one or more parameters are varied in small ranges. The coefficients of the asymptotic expansions are often called the sensitivities of the eigensolutions; they represent the (directional) derivatives of eigenvalues and eigenvectors with respect to the perturbation parameters. Some background on the perturbation theory of the eigenvalues can be found in the books by Wilkinson [1] and Kato [2].

Many papers have been devoted to eigensolutions sensitivity in which perturbation methods or equivalent methods of explicit differentiation have been employed. Most of them deal with self-adjoint systems; others with general systems. References can be found in the surveys by Adelman and Haftka [3] and Murthy and Haftka [4] as well as in the book by Brandon [5]. The problem of multiple eigenvalues has also been taken into account, but almost exclusively as regards self-adjoint systems (by Mills-Curran [6], Daily [7], and How and Kenny [8]) or non-defective, non-self-adjoint systems (Juang et al. [9]). Indeed, when a multiple eigenvalue occurs in a general system, its geometric multiplicity is often smaller than the algebraic multiplicity; so the (proper) eigenvectors of the system do not span the whole space, and generalized eigenvectors have to be introduced (e.g., see the book by Friedman [10]). Such systems are said to be defective. In mechanics, non-proportional damping or non-conservative effects (such as follower forces) are required in order for a system to be defective; in fact, conservative or proportionally damped systems always have a complete set of eigenvectors. 
Eigenderivatives of general defective systems have been studied by the author in reference [11] by utilizing perturbation methods; an application to a mechanical problem has also been presented in reference [12]. It has been shown there that the eigensolutions of defective systems are strongly sensitive to perturbations, since asymptotic expansions involve fractional powers of the perturbation parameter. A different approach to the problem was followed in the paper by Triantafyllou and Triantafyllou [13], in which a geometric theory was developed to explain the frequency coalescence phenomenon. In addition, a sensitivity analysis of simple conservative and dissipative systems was performed; however, no general computational methods for large systems analysis have been given.

Defective systems, however, represent exceptional cases. More often systems having close eigenvalues are encountered (mistuned systems), instead of perfectly coincident (tuned systems). In these cases, if the associated eigenvectors make groups of nearly parallel vectors, the system can be classified as nearly defective. If a standard procedure of sensitivity analysis for distinct eigenvalues is applied to such a system, series expansions are obtained, valid in very narrow regions of the space of the parameters, even if the mistuning is not very small; therefore they are useless for practical purposes.

This circumstance can easily be illustrated by a simple example. Consider the matrix

$$
\hat{A}(\xi)=\left[\begin{array}{ll}
1 & 1 \\
\xi & 1
\end{array}\right],
$$

depending on one small parameter $\xi \ll 1$. It admits two nearly coincident eigenvalues $\hat{\lambda}_{1,2}(\xi)=1 \pm \xi^{1 / 2}$ and two nearly parallel eigenvectors $\hat{u}_{1,2}(\xi)=\left\{1, \pm \xi^{1 / 2}\right\}^{\mathrm{T}}$; therefore it is nearly defective with mistuning $\xi^{1 / 2}$. Now perturb the matrix $\hat{A}$ as

$$
\tilde{A}(\xi, \eta)=\left[\begin{array}{cc}
1 & 1+\eta \\
\xi+\eta & 1
\end{array}\right],
$$

where $\eta \ll 1$ is a second small parameter. The eigenvalues of the perturbed matrix $\tilde{A}$ are $\tilde{\lambda}_{1,2}(\xi, \eta)=1 \pm[(1+\eta)(\xi+\eta)]^{1 / 2}$. By expanding them for small $\eta$ and keeping $\xi$ fixed the following expression is obtained:

$$
\tilde{\lambda}_{1,2}(\xi, \eta)=\hat{\lambda}_{1,2}(\xi) \pm \eta\left\{(1+\xi) / 2 \xi^{1 / 2}\right\}+O\left(\eta^{2}\right) .
$$

This expansion is not uniformly valid, since the second term is of the same order of or larger than the first term when $\eta \geqslant O\left(\xi^{1 / 2}\right)$.

The basic idea on how to approximate eigensolutions of perturbed nearly defective matrices $\tilde{A}$ is here borrowed from the theory of elastic stability by Koiter [14]. Accordingly, the unmodified nearly defective system $\hat{A}$ is viewed as a perfect (tuned) system $A_{0}$ affected by small imperfections causing the mistuning, controlled by a parameter $\xi$. When a second control parameter $\eta$ acts on the imperfect system (the modification parameter here, the load parameter in elastic stability), the solution is sought as a $\xi$-perturbation of the $\eta$-response of the perfect system, which itself is obtained by a perturbation method. However, Koiter's problem is a direct problem, since the perfect system $A_{0}$ is known; on the contrary, since only the imperfect system $\hat{A}$ is given here, a "close" ideal perfect system has to be found by solving an inverse problem.

If the previous example is considered again, a tuned system $A_{0}$ close to $\hat{A}$ can easily be found by posing $\xi=0$ in $\hat{A}(\xi)$. The matrix $A_{0}=\hat{A}(0)$ is a Jordan block admitting the double eigenvalue $\lambda_{0}=1$ and the unique proper eigenvector $u_{0}=\{1,0\}^{\mathrm{T}}$. If $A_{0}$ is perturbed only by $\eta$ (perturbed perfect system) the eigenvalues $\tilde{\lambda}_{1,2}(0, \eta)=1 \pm \eta^{1 / 2}+O\left(\eta^{3 / 2}\right)$ will be found. However, since $\xi$-perturbations are also present (perturbed imperfect system) the two 
parameters must be varied simultaneously. If a curve $\xi=\xi(\zeta), \eta=\eta(\zeta)$ is selected on the $(\xi, \eta)$-plane, the problem is conveniently converted into a one-parameter perturbation problem; by choosing the straight line $\xi=a \zeta, \eta=b \zeta$ and expanding $\tilde{\lambda}_{1,2}(\xi(\zeta), \eta(\zeta))$ around $\zeta=0$, it follows that

$$
\tilde{\lambda}_{1,2}(\xi, \eta)=1+[\zeta(a+b)]^{1 / 2}+O\left(\zeta^{3 / 2}\right)=1+(\xi+\eta)^{1 / 2}+O\left(\xi^{3 / 2}, \eta^{3 / 2}\right) .
$$

This expansion is uniformly valid. It can be considered to be a $\xi$-perturbation of $\tilde{\lambda}_{1,2}(0, \eta)$, to which it tends when $\eta \gg \xi$.

In this paper an algorithm for building up a suitable tuned system is developed for general systems in which the eigensolutions are nearly coincident in twos; however, the method can easily be extended to handle more general cases. After the ideal system is built up, a perturbation procedure similar to that utilized in reference [11] is followed, where, in addition, the mistuning is taken into account.

A simple two-dimensional system is considered first, then the mechanical two-d.o.f. (four-dimensional) system studied in reference [12] is analyzed.

\section{PERTURBATIONS OF GENERAL NEARLY DEFECTIVE SYSTEMS}

\subsection{DEFECTIVE SYSTEMS}

Consider an $N \times N$ real matrix $A=A(p)$, depending on vector $p$ of $M$ non-dimensional real parameters $p_{\alpha}(\alpha=1,2, \ldots, M)$. Suppose that in the $M$-dimensional space $\Pi$ of the parameters $p_{\alpha}$, a regular surface $\mathscr{S}$ having the following properties exists (see Figure 1). At any point $P \in \mathscr{S}$ there corresponds a matrix $A$ having a multiple eigenvalue $\lambda$ with algebraic multiplicity $m>1$ and geometric multiplicity $n=1$ (the general case $n<m$ dealt with in reference [11] will not be considered here); $u$ is the unique right eigenvector and $v$ is the unique left eigenvector $\left(A^{\mathrm{H}} v=\bar{\lambda} v\right)$ associated with $\lambda$. Therefore surface $\mathscr{S}$ represents a multi-parameter family of defective systems in the space $\Pi$.

Now fix a point $P_{0}$ on $\mathscr{S}$ and denote by $A_{0}=A\left(p_{0}\right)$ the corresponding matrix, having the multiple eigenvalue $\lambda_{0}$ and (unique) associated eigenvectors $u_{0}$ and $v_{0}$. It is well known that $m$ generalized right and left eigenvectors, $x_{i}$ and $y_{i}$, respectively, can be associated with the defective eigenvalue $\lambda_{0}$. They are determined by the recurrence formulas

$$
\left(A_{0}-\lambda_{0} I\right) x_{i}=x_{i-1}, \quad\left(A_{0}-\lambda_{0} I\right)^{\mathrm{H}} y_{i-1}=y_{i}, \quad i=2,3, \ldots, m,
$$

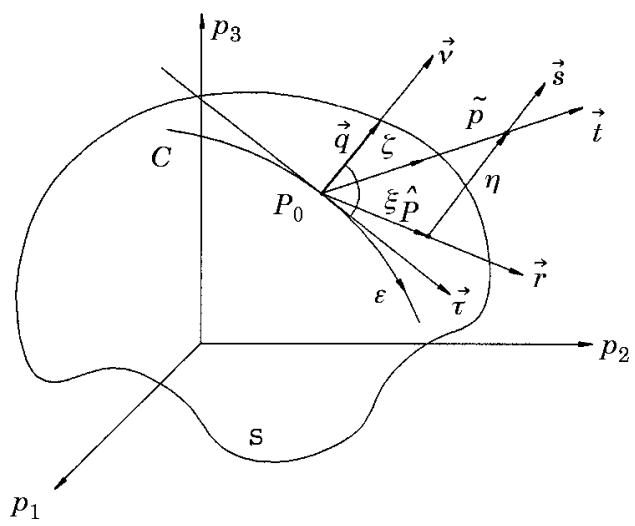

Figure 1. A multi-parameter family of defective systems and its perturbations in the parameter space. 
where $x_{1} \equiv u_{0}, y_{m} \equiv v_{0} ; i$ is called the index (or the order) of the eigenvector. The generalized eigenvectors are found from equations (5) to within arbitrary constants $a_{i}$ and $b_{i}$, since $x_{i}+a_{i} u_{0}$ and $y_{i}+b_{i} v_{0}$ are also solutions of the same equations. Constants $a_{i}$ can be chosen by imposing the normalization conditions $e_{h}^{\mathrm{T}} x_{1}=1, e_{h}^{\mathrm{T}} x_{j}=0(j=2,3, \ldots, m)\left(e_{h}=h\right.$ th $N$-dimensional canonical vector): i.e., by requiring that only the proper eigenvector $x_{1}$ has a non-vanishing $h$ th (properly selected) component. Constants $b_{i}$ can be determined by enforcing the bi-orthogonality conditions $y_{i}^{\mathrm{H}} x_{j}=\delta_{i j}$, some conditions being automatically verified as a consequence of equations (5).

\subsection{NEARLY DEFECTIVE SYSTEMS}

By perturbing the parameters $p_{\alpha}$ around $p_{\alpha 0}$ in the direction $\vec{r}=r_{\alpha} \vec{e}_{\alpha}$ (sum understood, $\vec{e}_{\alpha}=\alpha$ th $M$-dimensional canonical vector) (see Figure 1) it follows that $\hat{p}_{\alpha}(\xi)=p_{\alpha 0}+\xi r_{\alpha}$, $\xi= \pm\left\|P_{0} \hat{P}\right\| \ll 1$ being the perturbation parameter. Correspondingly, $\hat{A}(\xi)=A(\hat{p}(\xi))$ is expanded in a series of powers of $\xi$,

$$
\hat{A}(\xi)=A_{0}+\xi A_{\xi}+\frac{1}{2} \xi^{2} A_{\xi \xi}+\cdots,
$$

where

$$
A_{\xi}=\left(\partial A_{0} / \partial p_{\alpha}\right) r_{\alpha}, \quad A_{\xi \xi}=\left(\partial^{2} A_{0} / \partial p_{\alpha} \partial p_{\beta}\right) r_{\alpha} r_{\beta} .
$$

The unknown eigensolutions $\hat{\lambda}_{i}(\xi), \hat{u}_{i}(\xi)(i=1,2, \ldots, N)$ of $\hat{A}(\xi)$ can be determined through a perturbation procedure by assuming that they can be generated from the eigensolutions $\hat{\lambda}_{i}(0), \hat{u}_{i}(0)$ of the non-perturbed matrix $A_{0}$. However, while a standard procedure based on series expansions of integer powers of $\xi$ can be used for non-defective eigenvalues, series expansions of fractional powers of $\xi$ must be employed for defective eigenvalues (see the introductory example and references [1] and [11]). By limiting the attention to the $m$ eigenvalues generated by the defective eigenvalue $\lambda_{0}$, the following expansions are performed:

$$
\begin{gathered}
\hat{\lambda}_{k}(\xi)=\lambda_{0}+\xi^{1 / m} \dot{\lambda}_{0 k}+\frac{1}{2} \xi^{2 / m} \ddot{\lambda}_{0 k}+\cdots, \\
\hat{u}_{k}(\xi)=u_{0}+\xi^{1 / m} \dot{u}_{0 k}+\frac{1}{2} \xi^{2 / m} \ddot{u}_{0 k}+\cdots, \quad k=1,2, \ldots, m .
\end{gathered}
$$

Here the dot denotes differentiation with respect to $\xi^{1 / m}$. Moreover, to make the eigenvectors $\hat{u}_{k}$ unique, the normalization conditions $e_{h}^{\mathrm{T}} \hat{u}_{k}=1(k=1,2, \ldots, m)$ are enforced for any $\xi$, this entailing $e_{h}^{\mathrm{T}} \dot{u}_{0 k}=e_{h}^{\mathrm{T}} \ddot{u}_{0 k}=\cdots=0$.

The normalized coefficients of the series expansions (8) can be evaluated by solving the relevant perturbation equations and accounting for equations (5). It is found [11] that

$$
\begin{gathered}
\dot{\lambda}_{0 k}=\left(y_{m}^{\mathrm{H}} A_{\xi} x_{1}\right)^{1 / m}, \quad \ddot{\lambda}_{0 k}=2\left(y_{m}^{\mathrm{H}} A_{\xi} x_{2}-y_{m}^{\mathrm{H}} u_{*}\right) /\left(m \dot{\lambda}_{0 k}^{m-2}\right), \\
\dot{u}_{0 k}=\dot{\lambda}_{0 k} x_{2}, \quad \ddot{u}_{0 k}=\left\{\begin{array}{ll}
\ddot{\lambda}_{0 k} x_{2}+2 \dot{\lambda}_{0 k}^{2} x_{3} & \text { if } m>2 \\
\ddot{\lambda}_{0 k} x_{2}+2 u * & \text { if } m=2
\end{array}\right\},
\end{gathered}
$$

where $u_{*}$ is the (unique) solution of the non-homogeneous problem

$$
\left(A_{0}-\lambda_{0} I\right) u_{*}=\dot{\lambda}_{0 k}^{m} x_{m}-A_{\xi} x_{1}, \quad e_{h}^{\mathrm{T}} u_{*}=0 .
$$

Index $k$ in equations (9) and (10) refers to one of the $m$ complex roots furnished by equation (9a); $u_{*}$ does not depend on $k$.

Equations (8) and (9) show that a nearly defective system has nearly coincident eigenvalues $\hat{\lambda}_{k}$ and nearly parallel eigenvectors $\hat{u}_{k}$, since $\hat{\lambda}_{k}-\lambda_{0}=0\left(\xi^{1 / m}\right), \hat{u}_{k}-u_{0}=O\left(\xi^{1 / m}\right)$ for any $k$. The small quantity $\Delta=O\left(\xi^{1 / m}\right)$ will be referred to as the mistuning of the system. If left eigenvectors $\hat{v}_{k}$ are also of interest, they can be evaluated in the same way; it is found that 
$\hat{v}_{k}-v_{0}=O\left(\xi^{1 / m}\right)$ and $\hat{v}_{k}^{\mathrm{H}} \hat{u}_{k}=O\left(\xi^{1-1 / m}\right)$ : i.e., left eigenvectors are clustered around $v_{0}$ and are therefore nearly orthogonal to the corresponding right eigenvectors, clustered around $u_{0}$. Modal matrices $\hat{U}=\left[\hat{u}_{i}\right]$ and $\hat{V}=\left[\hat{v}_{i}\right]=\hat{U}^{-\mathrm{H}}(i=1,2, \ldots, N)$ are therefore ill-conditioned.

\subsection{SINGULAR PERTURBATIONS}

If $\dot{\lambda}_{0 k}=0$ the asymptotic expansions (8) are not valid. It is possible to show that this critical case occurs when the direction $\vec{r}$ of the perturbation is tangent to $\mathscr{S}$ at $P_{0}$.

To prove this property it is convenient first to re-write $\dot{\lambda}_{0 k}^{m} \equiv \dot{\lambda}_{0}^{m}$ as a scalar product, namely $\dot{\lambda}_{0}^{m}=\vec{q} \cdot \vec{r}$, where the vector $\vec{q}=v_{0}^{\mathrm{H}}\left(\partial A_{0} / \partial p_{\alpha}\right) u_{0} \vec{e}_{\alpha}$ has been defined and equations (7a) and (9a) have been used. Then, to find the direction of $\vec{q}$, one can proceed as follows.

Consider an arbitrary regular curve $C \in \mathscr{S}$ for $P_{0}$, having parametric equations $p_{\alpha}=p_{\alpha}(\epsilon)$, with $p_{\alpha}(0)=p_{\alpha 0}$ (see Figure 1). Along the curve $C, A=A(\epsilon), u=u(\epsilon), v=v(\epsilon)$, with $A(0)=A_{0}, u(0)=u_{0}$ and $v(0)=v_{0}$. As $A(\epsilon)$ is defective for any $\epsilon$, it ensues that $v^{\mathrm{H}}(\epsilon) u(\epsilon)=0$ or, from the eigenvalue problem, $v^{\mathrm{H}}(\epsilon) A(\epsilon) u(\epsilon)=0$. By differentiating the two identities with respect to $\epsilon$, and evaluating them at $\epsilon=0$, it follows that

$$
\dot{v}_{0}^{\mathrm{H}} u_{0}+v_{0}^{\mathrm{H}} \dot{u}_{0}=0, \quad \dot{v}_{0}^{\mathrm{H}} A_{0} u_{0}+v_{0}^{\mathrm{H}} A_{0} \dot{u}_{0}+v_{0}^{\mathrm{H}} \dot{A}_{0} u_{0}=0 .
$$

By virtue of equation (11a) and the eigenvalue problem, the sum of the first two terms in equations (11b) vanish and therefore $v_{0}^{\mathrm{H}} \dot{A}_{0} u_{0}=0$. Since $\dot{A}_{0}$ is proportional to $\left(\partial A_{0} / \partial p_{\alpha}\right) \tau_{\alpha}$, where $\tau_{\alpha}$ are the components of the unit vector $\vec{\tau}$ tangent to $C$ at $P_{0}$, from the previous result it ensues that $\vec{q} \cdot \vec{\tau}=0$; so, as $C$ is arbitrary, $\vec{q}$ is orthogonal to $\mathscr{S}$. Therefore, if the perturbation is tangent to $\mathscr{S}, \dot{\lambda}_{0 k}=0$, and if the perturbation is normal to $\mathscr{S}$, $\dot{\lambda}_{0 k}=\|\vec{q}\|^{1 / m}=\max$.

Tangent perturbations $(\vec{r} \equiv \vec{\tau})$ will be referred to as singular perturbations. When perturbations of the matrix $A_{0}$ are singular, the perturbations of the eigensolutions are of order smaller than $\xi^{1 / m}$; if $m=2$, the latter are of order $\xi$ : i.e., of the same order as the changes of the parameters. In the geometric theory of reference [13] such eigenvalue behaviour occurs near a degenerate coalescence point on the complex frequency plane.

\subsection{SENSITIVITIES OF NEARLY DEFECTIVE SYSTEMS}

If sensitivities of the eigensolutions $\hat{\lambda}_{k}, \hat{u}_{k}$ of a given nearly defective system $\hat{A}(\xi)$ have to be determined, parameters $p_{\alpha}$ must be perturbed around $\hat{p}_{\alpha}(\xi)$ (see Figure 1). A direction $\vec{s}=s_{\alpha} \vec{e}_{\alpha}$ is chosen and an abscissa $\eta= \pm\|\hat{P} \widetilde{P}\| \ll 1$ is introduced; so $\tilde{p}_{\alpha}(\eta ; \xi)=\hat{p}_{\alpha}(\xi)+\eta s_{\alpha}$ holds along $\vec{s}, \xi$ being a parameter. By expanding $\tilde{A}=A(\tilde{p}(\eta ; \xi))$ and its eigensolutions in series of integer powers of $\eta$ and keeping $\xi$ fixed, it follows that

$$
\begin{gathered}
\tilde{A}(\eta ; \xi)=\hat{A}+\eta \hat{A}_{\eta}+\frac{1}{2} \eta^{2} \hat{A}_{\eta \eta}+\cdots, \quad \tilde{\lambda}_{k}(\eta ; \xi)=\hat{\lambda}_{k}+\eta \hat{\lambda}_{k}^{\prime}+\frac{1}{2} \eta^{2} \hat{\lambda}_{k}^{\prime \prime}+\cdots, \\
\tilde{u}_{k}(\eta ; \xi)=\hat{u}_{k}+\eta \hat{u}_{k}^{\prime}+\frac{1}{2} \eta^{2} \hat{u}_{k}^{\prime \prime}+\cdots, \quad k=1,2, \ldots, m,
\end{gathered}
$$

where

$$
\hat{A}_{\eta}=\left(\partial \hat{A} / \partial p_{\alpha}\right) s_{\alpha}, \quad \hat{A}_{\eta \eta}=\left(\partial^{2} \hat{A} / \partial p_{\alpha} \partial p_{\beta}\right) S_{\alpha} S_{\beta}
$$

and the dash denotes differentiation with respect to $\eta$. It should be noted that expansions $(12 \mathrm{~b}, \mathrm{c})$ hold for any $k \in[1, N]$ since $\hat{A}$ is non-defective; however, attention will be focused here on nearly coincident eigensolutions only $(k \in[1, m])$. Equations $(12 \mathrm{~b}, \mathrm{c})$ will be referred to as standard perturbation expansions (SPE's). 
The following eigenvalues sensitivities are obtained:

$$
\begin{gathered}
\hat{\lambda}_{k}^{\prime}=\frac{\hat{v}_{k}^{\mathrm{H}} \hat{A}_{\eta} \hat{u}_{k}}{\hat{v}_{k}^{\mathrm{H}} \hat{u}_{k}}, \quad \hat{u}_{k}^{\prime}=\sum_{\substack{j=1 \\
j \neq k}}^{N} \frac{1}{\hat{\lambda}_{k}-\hat{\lambda}_{j}} \frac{\hat{v}_{j}^{\mathrm{H}} \hat{A}_{\eta} \hat{u}_{k}}{\hat{v}_{j}^{\mathrm{H}} \hat{u}_{j}} \hat{u}_{j}+a_{k} \hat{u}_{k}, \\
\hat{\lambda}_{k}^{\prime \prime}=\frac{\hat{v}_{k} \hat{A}_{\eta \eta} \hat{u}_{k}}{\hat{v}_{k}^{\mathrm{H}} \hat{u}_{k}}+\frac{2}{\hat{v}_{k}^{\mathrm{H}} \hat{u}_{k}} \sum_{\substack{j=1 \\
j \neq k}}^{N} \frac{1}{\hat{\lambda}_{k}-\hat{\lambda}_{j}} \frac{\hat{v}_{j}^{\mathrm{H}} \hat{A}_{\eta} \hat{u}_{k} \hat{v}_{k}^{\mathrm{H}} \hat{A}_{\eta} \hat{u}_{j}}{\hat{v}_{j}^{\mathrm{H}} \hat{u}_{j}}, \quad k=1,2, \ldots, m .
\end{gathered}
$$

Here $a_{k}$ is an arbitrary constant to be determined by the normalization conditions and $\hat{u}_{k}^{\prime}$ has been expanded in series of the eigenvectors $\hat{u}_{j}$. Since $\hat{\lambda}_{k}-\hat{\lambda}_{j}=O\left(\xi^{1 / m}\right)$ and $\hat{v}_{k}^{\mathrm{H}} \hat{u}_{k}=O\left(\xi^{1-1 / m}\right)$ for $k$ and $j \in[1, m]$, it ensues that $\tilde{\lambda}_{k}-\hat{\lambda}_{k}=O\left(\eta / \xi^{1-1 / m}\right)$ and $\tilde{u}_{k}-\hat{u}_{k}=O(\eta / \xi)$; therefore the SPE's are not uniformly valid when $\eta \geqslant O\left(\xi^{1-1 / m}\right)$ and $\eta \geqslant O(\xi)$, respectively.

Similar results have been obtained in reference [13] by analyzing series convergence instead of uniform validity. It is shown that a coalescence on the complex frequency plane maps into a branch point in the parameter space; therefore a power series expansion around a close regular point (i.e., around a nearly defective system) has a small radius of convergence, less than its distance from the singular point.

To obtain asymptotic expansions valid in a larger interval of $\eta$, the perturbation procedure is modified as follows. The eigensolutions $\tilde{\lambda}_{k}$ and $\tilde{u}_{k}$ are expanded in series of the two small parameters $\xi$ and $\eta$ around $\xi=\eta=0$; i.e., around the eigensolutions of the defective system $A_{0}$. The expansions are performed along the straight line $\vec{t}=t_{\alpha} \vec{e}_{\alpha}$ on which the abscissa $\zeta= \pm\left\|P_{0} \widetilde{P}\right\| \ll 1$ is introduced, so that $\xi \vec{r}+\eta \vec{s}=\vec{\zeta} \vec{t}$ (see Figure 1). By expanding $\tilde{A}(p(\zeta)$ ) it follows that

$$
\tilde{A}(\zeta)=A_{0}+\zeta A_{\zeta}+\frac{1}{2} \zeta^{2} A_{\zeta \zeta}+\cdots
$$

where

$$
A_{\zeta}=\left(\partial A_{0} / \partial p_{\alpha}\right) t_{\alpha}, \quad A_{\zeta \zeta}=\left(\partial^{2} A_{0} / \partial p_{\alpha} \partial p_{\beta}\right) t_{\alpha} t_{\beta}
$$

Since $A_{0}$ is defective, $\tilde{\lambda}_{k}(\zeta)$ and $\tilde{u}_{k}(\zeta)$ have to be expanded in series of $\zeta^{1 / m}$. So, expressions similar to equations (8) and (9) hold, by replacing $\xi$ by $\zeta$. By taking into account that $\zeta A_{\zeta}=\xi A_{\xi}+\eta A_{\eta}$, where $A_{\eta}=\left(\partial A_{0} / \partial p_{\alpha}\right) s_{\alpha}$, the dependence on the two parameters $\xi$ and $\eta$ can be made explicit. The following expressions are thus obtained:

$$
\tilde{\lambda}_{k}(\xi, \eta)=\lambda_{0}+\delta_{1} \lambda_{k}+\frac{1}{2} \delta_{2} \lambda_{k}+\cdots, \quad \tilde{u}_{k}(\xi, \eta)=u_{0}+\delta_{1} u_{k}+\frac{1}{2} \delta_{2} u_{k}+\cdots .
$$

Here

$$
\begin{gathered}
\delta_{1} \lambda_{k}=\left[y_{m}^{\mathrm{H}}\left(\xi A_{\xi}+\eta A_{\eta}\right) x_{1}\right]^{1 / m}, \quad \delta_{2} \lambda_{k}=2\left[y_{m}^{\mathrm{H}}\left(\xi A_{\xi}+\eta A_{\eta}\right) x_{2}-y_{m}^{\mathrm{H}} u *\right] /\left(m \delta_{1} \lambda_{k}^{m-2}\right), \\
\delta_{1} u_{k}=\delta_{1} \lambda_{k} x_{2}, \quad \delta_{2} u_{k}=\left\{\begin{array}{ll}
\delta_{2} \lambda_{k} x_{2}+2 \delta_{1} \lambda_{k}^{2} x_{3} & \text { if } m>2 \\
\delta_{2} \lambda_{k} x_{2}+2 u * & \text { if } m=2
\end{array}\right\},
\end{gathered}
$$

in which $u *$ is determined by

$$
\left(A_{0}-\lambda I\right) u_{*}=\delta_{1} \lambda_{k}^{m} x_{m}-\left(\xi A_{\xi}+\eta A_{\eta}\right) x_{1}, \quad e_{h}^{\mathrm{T}} u_{*}=0 .
$$

Equations (17) will be referred to as modified perturbation expansions (MPE's). It should be noted that they are not valid if the total perturbation $\vec{\zeta} \vec{t}$ is singular: i.e., if $\delta_{1} \lambda_{k}=0$. 


\section{THE INVERSE PROBLEM}

Practical problems can occur in the following form: a nearly defective system represented by a point $\hat{P}$ in the parameters space $\Pi$ is given and sensitivities of some nearly coincident eigenvalues and eigenvectors in the $\vec{s}$ direction have to be determined. To obtain MPE's a tuned defective system $P_{0}$ in the neighborhood of $\hat{P}$ must be selected as the starting point. However, in general systems points $P_{0}$ are not known and can be difficult to determine. Moreover, defective systems could not exist in $\Pi$, or at least only for meaningless values of the physical parameters; e.g., negative values of essentially positive quantities. So it appears convenient in any case to construct defective systems artfully.

The crucial task of how to build an ideal tuned system "close" to a real mistuned system constitutes an inverse problem. It is dealt with here under the simplifying assumption that only couples of nearly coincident eigenvalues occur (i.e., $m \leqslant 2$ ); a brief outline of how to handle more general cases is given in the Appendix.

In principle, the choice of the tuned system $P_{0}$ is arbitrary, but it should be expected that the approximation improves as $P_{0}$ becomes closer to $\hat{P}$. For this reason a good choice seems to be to search for an unknown tuned system $P_{0}$ that is transformed into the given mistuned system $\hat{P}$, after non-singular perturbation. The easiest way to accomplish this is to extend the $M$-dimensional space $\Pi=\left\{p_{\alpha}\right\}$ to an $(M+l)$-dimensional space $\Pi^{+}=\left\{p_{\alpha}, \xi_{j}\right\}$ by introducing suitable new parameters $\xi_{j}(j=1,2, \ldots, l)$ and to search for a defective system in the subspace $\hat{\Pi}^{+}=\left\{\hat{p}_{\alpha}, \xi_{j}\right\}$ of $\Pi^{+}$: i.e., leaving unchanged the values of the $M$ physical parameters $p_{\alpha}$. In other words, a perturbation matrix $-\delta A\left(\hat{p}_{\alpha}, \xi_{j}\left(\hat{p}_{\alpha}\right)\right)$ is added to $\hat{A}\left(\hat{p}_{\alpha}\right)$ so that a defective matrix $A_{0}\left(\hat{p}_{\alpha}, \xi_{j}\left(\hat{p}_{\alpha}\right)\right)$ is obtained.

An example for $N=2, M=1$ and $l=1$ is shown in Figure 2. In the space $\Pi$ (the $p_{1}$-axis), an isolated unknown defective system exists at the origin $O$, the distance of which from the given point $\hat{P}=\left(\hat{p}_{1}\right)$ is of the order of the mistuning $\Delta=\hat{\lambda}_{1}-\hat{\lambda}_{2}$ (i.e., the $p_{1}$-perturbations are singular). By introducing a suitably selected new parameter $\xi_{1}$, a one-parameter family of defective systems can be built up in the two-dimensional space $\Pi^{+}$. By choosing $P_{0}=\left(\hat{p}_{1}, \xi_{1}\left(\hat{p}_{1}\right)\right)$, a tuned system close to $\hat{P}$ is obtained, the distance of which from $\hat{P}$ is of order $\Delta^{2}$. Thus, in this case, extrapolating from $P_{0}$ (ideal defective system) appears to be more convenient than extrapolating from $O$ (real defective system).

By coming back to the general case, a matrix $\hat{A}$ with eigensolutions $\hat{\lambda}_{k}, \hat{u}_{k}$ and $\hat{v}_{k}$ $(k=1,2, \ldots, N)$ is known, with $\hat{v}_{i}^{\mathrm{H}} \hat{u}_{j}=\delta_{i j}$. Two cases are considered: (a) there is a unique couple of nearly coincident eigenvalues (with associated nearly parallel eigenvectors),

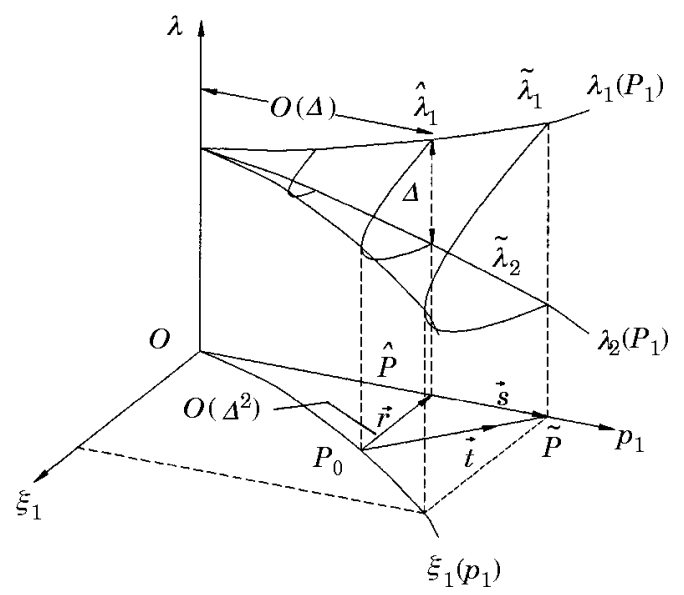

Figure 2. The ideal defective system $P_{0}$ in the extended parameters space. 
the remaining eigenvalues being well separated; (b) there are several couples of nearly coincident eigenvalues (each associated with nearly parallel eigenvectors) well separated among themselves and from the remaining eigenvalues. While sensitivities of separated eigensolutions can be determined by computing SPE's, sensitivities of nearly coincident eigensolutions must be evaluated by MPE's. To obtain them, and unknown defective matrix $A_{0}$ must be determined, so that $\|\delta A\|=\left\|\hat{A}-A_{0}\right\|$ is small with respect to $\|\hat{A}\|$.

\subsection{SINGLE COUPLE OF NEARLY COINCIDENT EIGENVALUES}

Let $\hat{\lambda}_{1}$ and $\hat{\lambda}_{2}$ be the (real) nearly coincident eigenvalues, with $\hat{\lambda}_{1}-\hat{\lambda}_{2}=O\left(\xi^{1 / 2}\right)$, $\xi$ being a small parameter. The space $\Pi=\left\{p_{\alpha}\right\}$ is extended by considering $\xi$ as the $(M+1)$ th (real) parameter. In the extended space $\Pi^{+}=\left\{p_{\alpha}, \xi\right\}$ a defective matrix $A_{0}$ is assumed to exist having the spectral representation $A_{0}=X J Y^{\mathrm{H}}$, where

$$
J=\left[\begin{array}{cc|ccc}
\lambda_{0} & 1 & & \\
& \lambda_{0} & & \\
\hline & & \lambda_{03} & \\
& & & \ddots & \\
& & & \lambda_{0 N}
\end{array}\right]
$$

contains a Jordan block with a double eigenvalue $\lambda_{0}$ and $X=\left[x_{k}\right]$ and $Y=\left[y_{k}\right]=X^{-\mathrm{H}}$ are the right and left eigenvector matrices, respectively. Only $x_{2}$ and $y_{1}$ are generalized (index two) eigenvectors, the remainder being proper eigenvectors. (The change of notation with respect to the previously defined eigenvectors (equations (5)), all associated with $\lambda_{0}$, should be noted.)

The perturbation matrix is written as $\delta A=\xi A_{\xi}$ and $A_{\xi}=\left[a_{i j}\right]$ is represented as an $N^{2}$-term dyad: i.e., $A_{\xi}=a_{i j} x_{i} y_{j}^{\mathrm{H}}$ (sum understood). If $\delta A$ has to be a non-singular perturbation of $A_{0}$, from equations ( $9 \mathrm{a}) y_{2}^{\mathrm{H}} A_{\xi} x_{1} \neq 0$ must hold: i.e., $a_{21} \neq 0$. The simplest choice is to assume that $a_{i j}=\delta_{i 2} \delta_{j 1}$ : i.e.,

$$
A_{\xi}=x_{2} y_{1}^{\mathrm{H}} .
$$

In other words, matrix $A_{0}$ is perturbed by replacing the zero $(2,1)$ coefficient of $J$ by $\xi$.

The perturbed matrix has the spectral representation $A_{0}+\xi A_{\xi}=\hat{U}_{0} \hat{\Lambda}_{0} \hat{V}_{0}^{\mathrm{H}}$, where

$$
\begin{gathered}
\hat{U}_{0}=\left[x_{1}+\xi^{1 / 2} x_{2}, x_{1}-\xi^{1 / 2} x_{2}, x_{3}, \ldots, x_{N}\right], \\
\hat{V}_{0}=\left[\left(y_{1}+\xi^{-1 / 2} y_{2}\right) / 2,\left(y_{1}-\xi^{-1 / 2} y_{2}\right) / 2, y_{3}, \ldots, y_{N}\right], \\
\hat{\Lambda}_{0}=\operatorname{diag}\left(\lambda_{0}+\xi^{1 / 2}, \lambda_{0}-\xi^{1 / 2}, \lambda_{03}, \ldots, \lambda_{0 N}\right) .
\end{gathered}
$$

It should be noted incidentally that the first two (normalized) left eigenvectors tend to infinity when $\xi \rightarrow 0$, because they are nearly orthogonal to the corresponding right eigenvectors.

The spectral representation of the known matrix is $\hat{A}=\hat{U} \hat{\Lambda} \hat{V}^{\mathrm{H}}$, where $\hat{U}=\left[\hat{u}_{k}\right], \hat{V}=\left[\hat{v}_{k}\right]$ and $\hat{\Lambda}=\operatorname{diag}\left(\hat{\lambda}_{k}\right)$. By equating the two matrices, i.e., by requiring $\hat{U}_{0}=\hat{U}, \hat{V}_{0}=\hat{V}$ and $\hat{\Lambda}_{0}=\hat{\Lambda}$, and solving with respect to the unknown quantities, it is found that

$$
\begin{aligned}
& \xi^{1 / 2}=\frac{1}{2}\left(\hat{\lambda}_{1}-\hat{\lambda}_{2}\right), \quad \lambda_{0}=\frac{1}{2}\left(\hat{\lambda}_{1}+\hat{\lambda}_{2}\right), \quad x_{1}=\frac{1}{2}\left(\hat{u}_{1}+\hat{u}_{2}\right), \quad x_{2}=\frac{1}{2} \xi^{-1 / 2}\left(\hat{u}_{1}-\hat{u}_{2}\right), \\
& y_{1}=\hat{v}_{1}+\hat{v}_{2}, \quad y_{2}=\xi^{1 / 2}\left(\hat{v}_{1}-\hat{v}_{2}\right) \text {, }
\end{aligned}
$$

together with $\lambda_{k}=\hat{\lambda}_{k}, x_{k}=\hat{u}_{k}$ and $y_{k}=\hat{v}_{k}$ for $k>2$.

It is worth noting that perturbations (21) couple only the first two eigensolutions of $A_{0}$, while they leave the other eigensolutions unaltered. In particular, $\xi, \lambda_{0}, x_{i}$ and $y_{i}(i=1,2)$ depend only on $\hat{\lambda}_{i}, \hat{u}_{i}$ and $\hat{v}_{i}$. Therefore, if only the sensitivities of the two nearly coincident 
eigensolutions are of interest, then the remaining eigensolutions of $\hat{A}$ do not have to be evaluated.

In conclusion, the following algorithm can be applied.

(1) From the eigenvalue problems $(\hat{A}-\hat{\lambda} I) \hat{u}=0$ and $(\hat{A}-\hat{\lambda} I)^{\mathrm{H}} \hat{v}=0, \hat{\lambda}_{i}, \hat{u}_{i}$ and $\hat{v}_{i}$ $(i=1,2)$ are evaluated; right eigenvectors $\hat{u}_{i}$ are normalized according to $e_{h}^{\mathrm{T}} \hat{u}_{i}=1$ and left eigenvectors to $\hat{v}_{i}^{\mathrm{H}} \hat{u}_{j}=\delta_{i j}$.

(2) Equations (23) are employed to calculate $\xi, \lambda_{0}, x_{i}$ and $y_{i}(i=1,2)$ (the normalization condition $e_{h}^{\mathrm{T}} x_{i}=1$ being automatically satisfied).

(3) Matrix $A_{\xi}$ is computed from equation (21) and matrix $A_{0}$ is then obtained as a difference, $A_{0}=\hat{A}-\xi x_{2} y_{1}^{\mathrm{H}}$.

(4) For a given modification matrix $A_{\eta}$, the modified eigensolutions $\tilde{\lambda}_{i}$ and $\tilde{u}_{i}$ are calculated from equations (17)-(19) (with $m=2$ ) as functions of a parameter $\eta$, $\xi$ being fixed. Namely:

$$
\begin{gathered}
\tilde{\lambda}_{i}(\xi, \eta)=\lambda_{0} \pm\left(\xi+\eta y_{2}^{\mathrm{H}} A_{\eta} x_{1}\right)^{1 / 2}+\frac{1}{2} \eta\left(y_{2}^{\mathrm{H}} A_{\eta} x_{2}-y_{2}^{\mathrm{H}} u *\right), \\
\tilde{u}_{i}(\xi, \eta)=u_{0} \pm\left(\xi+\eta y_{2}^{\mathrm{H}} A_{\eta} x_{1}\right)^{1 / 2} x_{2}+\eta\left[\frac{1}{2}\left(y_{2}^{\mathrm{H}} A_{\eta} x_{2}-y_{2}^{\mathrm{H}} u_{*}\right) x_{2}+u_{*}\right], \quad i=1,2,
\end{gathered}
$$

where $u *$ is the solution of

$$
\left(A_{0}-\lambda I\right) u_{*}=\left(y_{2}^{\mathrm{H}} A_{\eta} x_{1}\right) x_{2}-A_{\eta} x_{1}, \quad e_{h}^{\mathrm{T}} u_{*}=0 .
$$

Equations (24) and (25) are simpler than general equations (17)-(19), since the squared mistuning $\xi$ appears only at the first order; this is due to the particularly simple form (21) of $A_{\xi}$. MPE's performed by using points $P_{0} \in \Pi$ as starting points do not generally have this property. There are two consequences as a result of this: (a) when $\eta \rightarrow 0$ second order MPE's furnish the exact solution; (b) the vector $u *$ can be made independent of $\eta$, so it can be calculated once and for all. The last circumstance ensures the effectiveness of the method; if otherwise, it would not be computationally convenient.

\subsection{SEVERAL COUPLES OF NEARLY COINCIDENT EIGENVALUES}

Let $\hat{\lambda}_{1}, \hat{\lambda}_{2}, \ldots, \hat{\lambda}_{2 l}$ be $2 l$ (generally complex) eigenvalues, nearly coincident in twos, with $\hat{\lambda}_{1}-\hat{\lambda}_{2}=O\left(\xi_{1}^{1 / 2}\right), \hat{\lambda}_{3}-\hat{\lambda}_{4}=O\left(\xi_{2}^{1 / 2}\right), \ldots, \hat{\lambda}_{2 l-1}-\hat{\lambda}_{2 l}=O\left(\xi_{l}^{1 / 2}\right)$, where $\xi_{1}, \xi_{2}, \ldots, \xi_{l}$ are small parameters. The space $\Pi$ is extended by considering the $\xi_{j}$ 's as additional (generally complex) parameters. In the $(M+l)$-dimensional space $\Pi^{+}$a defective matrix $A_{0}$ is sought. Its Jordan canonical form contains $l$ blocks having double eigenvalues $\lambda_{01}, \lambda_{03}, \ldots, \lambda_{0,2 l-1}$, so that $x_{1}, x_{3}, \ldots, x_{2 l-1}, x_{k}(k>2 l)$ are proper eigenvectors and $x_{2}, x_{4}, \ldots, x_{2 l}$ are generalized (index two) right eigenvectors. Similarly, $y_{2}, y_{4}, \ldots, y_{2 l}, y_{k}(k>2 l)$ are proper left eigenvectors and $y_{1}, y_{3}, \ldots, y_{2 l-1}$ are generalized left eigenvectors.

The following perturbation matrix $\delta A$ is chosen:

$$
\delta A=\xi_{1} A_{\xi_{1}}+\xi_{2} A_{\xi_{2}}+\cdots+\xi_{l} A_{\xi_{l}}=\xi_{1} x_{2} y_{1}^{\mathrm{H}}+\xi_{2} x_{4} y_{3}^{\mathrm{H}}+\cdots+\xi_{l} x_{2 l} y_{2 l-1}^{\mathrm{H}} .
$$

Accordingly, the $l$ Jordan blocks are perturbed by posing the $(2,1)$ coefficients equal to $\xi_{j}$ $(j=1,2, \ldots, l)$. As previously noted, such a perturbation couples only the eigenvectors of each block, not the eigenvectors of different blocks. Therefore, by proceeding as in the previous case, formulas similar to equations (23) are derived,

$$
\begin{array}{ll}
\xi_{j}^{1 / 2}=\frac{1}{2}\left(\hat{\lambda}_{2 j-1}-\hat{\lambda}_{2 j}\right), \quad \lambda_{0,2 j-1}=\frac{1}{2}\left(\hat{\lambda}_{2 j-1}+\hat{\lambda}_{2 j}\right), & x_{2 j-1}=\frac{1}{2}\left(\hat{u}_{2 j-1}+\hat{u}_{2 j}\right), \\
x_{2 j}=\frac{1}{2} \xi_{j}^{-1 / 2}\left(\hat{u}_{2 j-1}-\hat{u}_{2 j}\right), \quad y_{2 j-1}=\hat{v}_{2 j-1}+\hat{v}_{2 j}, & y_{2 j}=\xi_{j}^{1 / 2}\left(\hat{v}_{2 j-1}-\hat{v}_{2 j}\right),
\end{array}
$$

whereas $\lambda_{0 k}=\hat{\lambda}_{k}, x_{k}=\hat{u}_{k}$ and $y_{k}=\hat{v}_{k}(k>2 l)$ are still obtained.

By referring to the $j$ th couple of nearly coincident eigensolutions, modified eigensolutions $\tilde{\lambda}_{i}$ and $\tilde{u}_{i}(i=2 j-1,2 j)$ can be obtained by equations (17)-(19) by replacing $\xi A_{\xi}$ by $\delta A=\Sigma_{j=1}^{l} \xi_{j} A_{\xi_{j}}$ and reading $x_{2 j-1}, x_{j}, y_{2 j-1}$ and $y_{2 j}$ instead of $x_{1}, x_{2}, y_{1}$ and $y_{2}$. 
However, due to the choice (26) of $\delta A$ and the bi-orthogonality conditions among the generalized eigenvectors, only the perturbation $\xi_{j} A_{\xi_{j}}$ gives non-vanishing contributions to $\tilde{\lambda}_{i}$ and $\tilde{u}_{i}$. In conclusion, the algorithm valid for a single couple of nearly coincident eigenvalues is still valid for several couples, provided that the couples are well separated. So the analysis can be performed singularly for each couple by applying equations (24) and (25) and ignoring the other couples. However, if second order sensitivities $\delta_{2} \lambda_{i}$ and $\delta_{2} u_{i}$ have to be evaluated, matrix $A_{0}=\hat{A}-\Sigma_{j=1}^{l} \xi_{j} A_{\xi_{j}}$ must be evaluated to find $u_{*}$, thus entailing the necessity to calculate all the right and left eigenvectors associated with the l couples of eigenvalues.

It should be noted from equations (27) that the parameters $\xi_{j}$ and the eigenvectors of $A_{0}$ associated with complex conjugate eigenvalues of $\hat{A}$ themselves are complex conjugate, so that, from equation (26), $\delta A$ is real, and therefore $A_{0}$ is also real.

An important particular case occurs when $l=2$ and the two couples of eigenvalues are complex conjugate. Then $\xi_{1} \equiv \xi, \xi_{2} \equiv \bar{\xi}$ and equation (26) reads

$$
\delta A=2 \operatorname{Re}\left[\xi x_{2} y_{1}^{\mathrm{H}}\right] .
$$

\section{EXAMPLE 1: A TWO DIMENSIONAL SYSTEM}

As a first example, consider the free motion $z(t)$ of a damped, single-d.o.f. system, governed by the differential equation $\ddot{z}+2 \delta \dot{z}+\kappa z=0$. Upon assuming $z=\{\dot{z}, z\}^{\mathrm{T}}$ as the state vector, the dynamical matrix is

$$
A=\left[\begin{array}{cc}
-2 \delta & -\kappa \\
1 & 0
\end{array}\right] .
$$

The system is two-dimensional $(N=2)$ and depends on two physical parameters $(M=2)$ : i.e., $p=\left\{p_{1}, p_{2}\right\} \equiv\{\delta, \kappa\}^{\mathrm{T}}$. It admits the following eigenvalues $\lambda_{k}$ and right and left bi-orthgonal normalized eigenvectors $u_{k}$ and $v_{k}(k=1,2)$ :

$$
\lambda_{1,2}=-\delta \pm \sqrt{\delta^{2}-\kappa}, \quad u_{1,2}=\left\{\lambda_{1,2}, 1\right\}^{\mathrm{T}}, \quad v_{1,2}=\left\{-\lambda_{1,2}, \kappa\right\}^{\mathrm{T}} /\left(\kappa-\lambda_{1,2}^{2}\right) .
$$

The eigenvalues $\lambda_{1,2}$ are represented in Figure 3 versus the parameters $p_{\alpha}$.

Along the curve $C$ of equation $\kappa=\delta^{2}$ the system is defective (critically damped). In fact, it has a unique double eigenvalue $\lambda_{0}=-\delta(m=2, n=1)$ and unique right and left eigenvectors, $u_{0}=\{-\delta, 1\}^{\mathrm{T}}$ and $v_{0}=\{\delta, \kappa\}$ respectively; in addition, $v_{0}^{\mathrm{H}} u_{0}=0$.
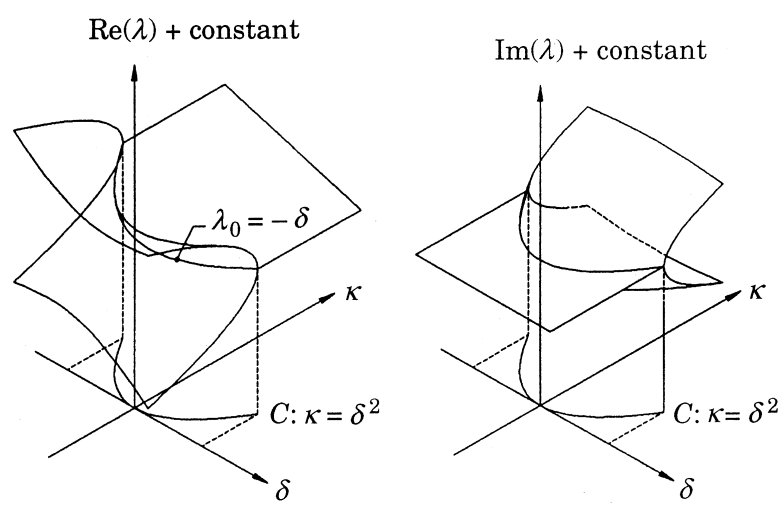

Figure 3. Eigenvalues loci for the system (29). 


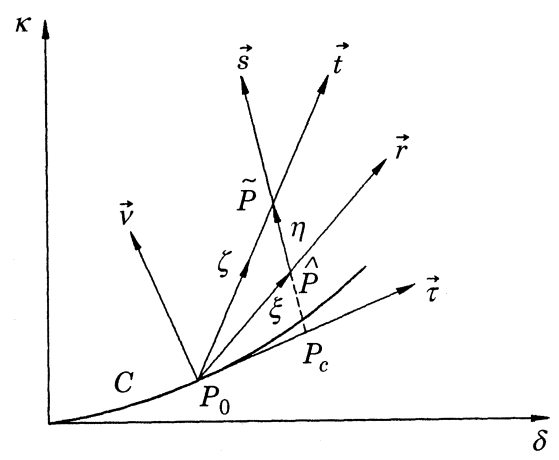

Figure 4. Parameters space for the system (29).

By perturbing a selected point $P_{0} \in C$ in the direction $\vec{r}=r_{\delta} \vec{e}_{\delta}+r_{\kappa} \vec{e}_{\kappa}$, nearly defective systems $\hat{P}$ are obtained (see Figure 4), with parameters $\hat{\delta}=\delta_{0}+\xi r_{\delta}$ and $\hat{\kappa}=\kappa_{0}+\xi r_{k}$. Their eigensolutions expansions could be found by applying general equations (8)-(10); however, since closed form solutions (30) are known in this case, it is simpler to expand equations (30) directly in series of $\xi$. The following $\xi^{1 / 2}$-derivatives are obtained:

$$
\begin{gathered}
\dot{\lambda}_{0 k}= \pm\left(2 \delta_{0} r_{\delta}-r_{k}\right)^{1 / 2}, \quad \ddot{\lambda}_{0 k}=-2 r_{\delta}, \quad \lambda_{0 k}^{(3)}=3 r_{\delta}^{2} / \dot{\lambda}_{0 k}, \\
\dot{u}_{0 k}=\left\{\dot{\lambda}_{0 k}, 0\right\}^{\mathrm{T}}, \quad \ddot{u}_{0 k}=\left\{\ddot{\lambda}_{0 k}, 0\right\}^{\mathrm{T}}, \quad u_{0 k}^{(3)}=\left\{\lambda_{0 k}^{(3)}, 0\right\}^{\mathrm{T}}, \quad k=1,2 .
\end{gathered}
$$

When $\vec{r}$ is varied, with $P_{0}$ remaining unchanged, the sensitivity of the eigensolutions varies. By observing that $\dot{\lambda}_{0 k}= \pm\left[-\left(1+4 \delta_{0}^{2}\right)^{1 / 2} \vec{r} \cdot \vec{v}\right]^{1 / 2}$, where $\vec{v}$ is the unit vector normal to $C$ at $P_{0}$, it follows that the greatest sensitivity occurs when perturbations are normal to $C(\vec{r} \equiv \vec{v})$, and the smallest sensitivity when the parameters are perturbed in the tangential direction $(\vec{r} \equiv \vec{\tau})$. In the last case (singular perturbations) $\dot{\lambda}_{0 k}=0$ and sensitivity is of order $\xi$, instead of $\xi^{1 / 2}$; in addition, expansions (8) cannot be applied, since $\lambda_{0 k}^{(3)}=\infty$. The region of non-uniformity of the asymptotic expansions (8) is $\vec{r} \cdot \vec{v} \leqslant O(\xi)$, as ensues by equating the orders of the different terms. For tangent perturbations an exact linear variation of $\lambda$ is found: i.e., $\hat{\lambda}_{k}=-\delta_{0}+(-1 \pm 1) \xi r_{\delta}$.

By perturbing $\hat{P}$ again, systems $\widetilde{P}$ along $\vec{s}$ are obtained (see Figure 4$)$. By posing $\tilde{\delta}=\hat{\delta}+\eta s_{\delta}$ and $\tilde{\kappa}=\hat{\kappa}+\eta s_{\kappa}$ and expanding equations (30) for small $\eta$ and $\xi$ fixed, equations (12b, c) are obtained, with

$$
\begin{gathered}
\hat{\lambda}_{k}^{\prime}=-s_{\delta} \pm\left(2 \hat{\delta} s_{\delta}-s_{k}\right) /(2 \Delta), \quad \hat{\lambda}_{k}^{\prime \prime}= \pm s_{\delta}^{2} / \Delta \mp\left(2 \hat{\delta} s_{\delta}-s_{k}\right)^{2} /\left(4 \Delta^{3}\right), \\
\hat{u}_{k}^{\prime}=\left\{\hat{\lambda}_{k}^{\prime}, 0\right\}^{\mathrm{T}}, \quad \hat{u}_{k}^{\prime \prime}=\left\{\hat{\lambda}_{k}^{\prime \prime}, 0\right\}^{\mathrm{T}}, \quad k=1,2,
\end{gathered}
$$

and $\Delta=\Delta(\xi)=\left(\hat{\lambda}_{1}-\hat{\lambda}_{2}\right) / 2=\left(\delta^{2}-\kappa\right)^{1 / 2}$. Analogous expressions hold for left eigenvectors. Parameter $\Delta \ll 1$ is a mistuning parameter, accounting for the small differences between the eigenvalues; namely, $\Delta=O\left(\xi^{1 / 2}\right)$ if $\vec{r} \neq \vec{\tau}$ and $\Delta=O(\xi)$ if $\vec{r} \equiv \vec{\tau}$. As a consequence, series $(12 \mathrm{~b}, \mathrm{c})$ are valid only for very small $\eta$. In fact, when $\eta=O\left(\Delta^{2}\right)$ the third term comes to be of the same order as the second, and when $\eta=O(\Delta)$ the second term comes to be of the same order as the first. Therefore equations $(12 \mathrm{~b}, \mathrm{c})$ cannot be used in practice.

One can now perform MPE's by assuming $P_{0} \in C$ as the starting point (i.e., without extending the space $\Pi$ ). The following $\zeta^{1 / 2}$-derivatives in the $\vec{t}$ direction (see Figure 4 ) are obtained:

$$
\begin{gathered}
\dot{\lambda}_{0 k}= \pm\left(2 \delta_{0} t_{\delta}-t_{k}\right)^{1 / 2}, \quad \ddot{\lambda}_{0 k}=-2 t_{\delta}, \quad \lambda_{0 k}^{(3)}=3 t_{\delta}^{2} / \dot{\lambda}_{0 k}, \\
\dot{u}_{0 k}=\left\{\dot{\lambda}_{0 k}, 0\right\}^{\mathrm{T}}, \quad \ddot{u}_{0 k}=\left\{\ddot{\lambda}_{0 k}, 0\right\}^{\mathrm{T}}, \quad u_{0 k}^{(3)}=\left\{\lambda_{0 k}^{(3)}, 0\right\}^{\mathrm{T}}, \quad k=1,2 .
\end{gathered}
$$


Upon recalling that $\xi t_{\delta}=\xi r_{\delta}+\eta s_{\delta}$ and $\xi t_{\kappa}=\xi r_{\kappa}+\eta s_{\kappa}$, it follows that

$$
\begin{aligned}
\tilde{\lambda}_{1,2}(\xi, \eta)= & -\delta_{0} \pm\left[\xi\left(2 \delta_{0} r_{\delta}-r_{\kappa}\right)+\eta\left(2 \delta_{0} s_{\delta}-s_{\kappa}\right)\right]^{1 / 2}-\left(\xi r_{\delta}+\eta s_{\delta}\right) \\
& \pm\left(\xi r_{\delta}+\eta s_{\delta}\right)^{2} / 2\left[\xi\left(2 \delta_{0} r_{\delta}-r_{\kappa}\right)+\eta\left(2 \delta_{0} s_{\delta}-s_{\kappa}\right)\right]^{1 / 2} .
\end{aligned}
$$

The asymptotic expansion (34) is not affected by the drawback of expansions (12b); however, it has a narrow region of non-uniformity around $\vec{t}=\vec{\tau}$ : i.e., for total perturbations $\vec{\zeta} \vec{t}$ of singular type, as occurs for point $P_{c} \in s$ in Figure 4.

If the algorithm of Section 3 is applied instead, the following results are obtained,

$$
\begin{aligned}
\xi=\hat{\delta}^{2}-\hat{\kappa}, & \lambda_{0}=-\hat{\delta}, \quad X=\left[\begin{array}{cc}
-\hat{\delta} & 1 \\
1 & 0
\end{array}\right], \quad Y=\left[\begin{array}{ll}
0 & 1 \\
1 & \hat{\delta}
\end{array}\right], \\
A_{0} & =\left[\begin{array}{cc}
-2 \hat{\delta} & -\hat{\delta}^{2} \\
1 & 0
\end{array}\right], \quad u_{*}=0,
\end{aligned}
$$

and, from equation (20a), it follows that

$$
\tilde{\lambda}_{1,2}=-\hat{\delta} \pm\left[\delta^{2}-\hat{\kappa}+\eta\left(2 \hat{\delta} s_{\delta}-s_{\kappa}\right)\right]^{1 / 2}-\eta s_{\delta} .
$$

So, in this particular example, it is easy to check that extrapolating from $P_{0}^{+}=(\hat{\delta}, \hat{\kappa}, \xi)$ in the extended three-dimensional $\Pi^{+}$space is equivalent to extrapolating from $P_{0}=\left(\hat{\delta}, \hat{\delta}^{2}\right)$ in the $\Pi$ space.

Two numerical examples have been worked out to compare exact, SPE's and MPE's. SPE's were truncated to second order $\left(\eta^{2}\right)$ terms; and MPE's to third order $\left(\zeta^{3 / 2}\right)$ or second order $\left(\zeta^{2 / 2}\right)$ terms. A nearly defective system $\hat{P}$ was given and eigenvalues sensitivities in a selected $\vec{s}$ direction were evaluated. The MPE (equation (34)) was built up starting from a point $P_{0}$ close to $\hat{P}$, arbitrarily chosen on curve $C$.

As a first example, $\hat{P}=(1,0 \cdot 9)$ and $s_{\delta}=-s_{\kappa}=\sqrt{2} / 2$ were considered. By varying $\eta$ in the interval $[-0 \cdot 5,0 \cdot 5]$, the eigenvalues loci shown in Figure 5 were obtained. The SPE is a very poor approximation of the exact solution; in particular, it furnishes $\operatorname{Im}(\tilde{\lambda})=0$ for any $\eta$. On the contrary, the third order MPE relative to the starting point $P_{0}=(1,1)$ and
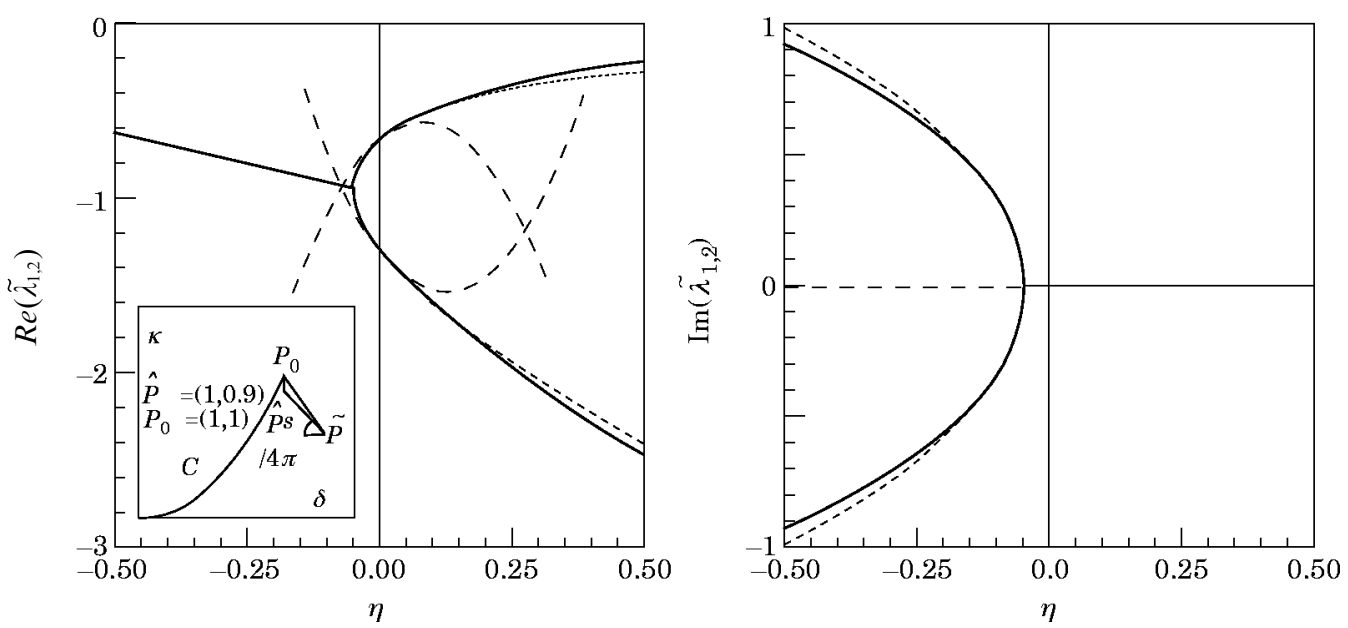

Figure 5. Eigenvalues versus modifications for $\hat{\delta}=1, \hat{\kappa}=0.9$ and $s_{\delta}=-s_{\kappa}=\sqrt{2} / 2$. - Exact; —-, third order MPE; --, SPE; - - -, second order MPE. 

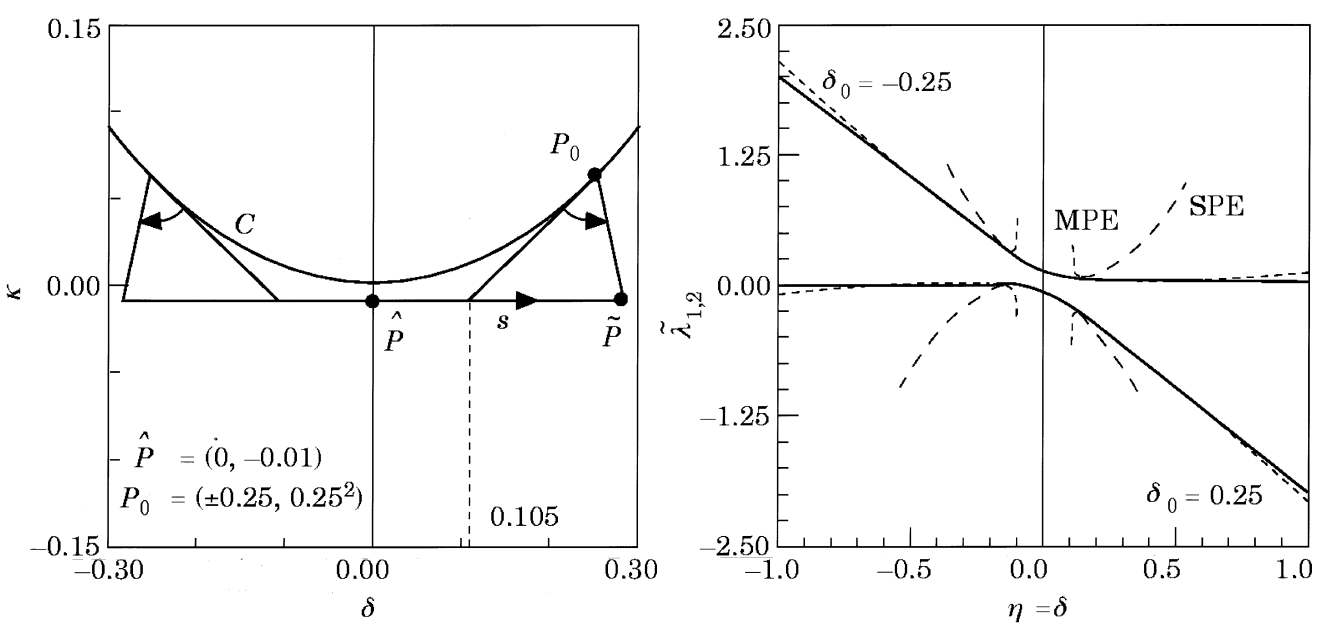

Figure 6. Parameters space and eigenvalues versus modifications for $\hat{\delta}=0, \hat{\kappa}=-0 \cdot 01, s_{\delta}=1$ and $s_{\kappa}=0 .-$, Exact; --, SPE; - - - , third order MPE.

the exact solution are undistinguishable in the plot, although a large interval of $\eta$ is considered; the second order approximation also furnishes an excellent approximation. In this case the choice of point $P_{0}$ does not substantially affect the solution.

As a second example, $\hat{P}=(0,-0 \cdot 01)$ and $s_{\delta}=1, s_{\kappa}=0$ were considered, so that $\eta \equiv \delta$ (see Figure 6(a)). The eigenvalues $\tilde{\lambda}_{k}$ are real for any $\eta$ and are plotted in Figure 6(b). As in the previous case, the SPE rapidly diverges from the exact solution. Two MPE's were computed, starting from two different points of $C$, namely $P_{0}=\left( \pm 0 \cdot 25,0 \cdot 25^{2}\right)$. The two solutions go to $\pm \infty$ when $\eta= \pm 0 \cdot 105$ (see Figure 6(a)), but furnish good approximations of the exact solution on one side of the asymptotes. This is due to the fact that the parameters are perturbed along a direction $\vec{s} \equiv \vec{e}_{\delta}$, forming a small angle with the tangents $\vec{\tau}$ to $C$ at points $P_{0}$ near the origin, so they have the character of singular perturbations. In this critical case, therefore, the choice of $P_{0}$ appears to be important.

\section{EXAMPLE 2: A FOUR-DIMENSIONAL SYSTEM}

As a second example consider the two-d.o.f. system studied in reference [12], consisting of two single-d.o.f. oscillators of stiffness $k_{i}$ and mass $m_{i}(i=1,2)$, coupled by a damper of constant $c$. With the state vector denoted by $z=\left\{\dot{z}_{1}, \dot{z}_{2}, z_{1}, z_{2}\right\}^{\mathrm{T}}, z_{i}$ being the displacement of the oscillator $i$ and $\dot{z}_{i}$ its velocity, the dynamic matrix of the system is

$$
A=\left[\begin{array}{ccrc}
-2 \delta & 2 \delta & -1 & 0 \\
2 \delta / \mu & -2 \delta / \mu & 0 & -\kappa / \mu \\
1 & 0 & 0 & 0 \\
0 & 1 & 0 & 0
\end{array}\right]
$$

where $\mu=m_{2} / m_{1}, \kappa=k_{2} / k_{1}$ and $2 \delta=c / \sqrt{k_{1} m_{1}}$ are non-dimensional parameters. The system is therefore four-dimensional $(N=4)$ and the space $\Pi$ three-dimensional $(M=3)$, since $p=\{\kappa, \mu, \delta\}^{\mathrm{T}}$. It is easy to check that if

$$
\mu=(1 \pm \delta) /(1 \mp \delta), \quad \kappa=1 / \mu,
$$

matrix $A$ is defective, since it admits two double eigenvalues (complex conjugate if $\delta<\sqrt{2} / 2)$, each associated with a unique eigenvector $(m=2, n=1)$. Equations (35) define 
two curves, $C_{1}$ and $C_{2}$, in $\Pi$ : i.e., two one-parameter families of defective systems (see Figure 7). Due to the symmetry of the system, one family can be obtained from the other simply by exchanging stiffnesses and masses of the oscillators.

Now one can consider a nearly defective system $\hat{A}$, represented in the space $\Pi$ by a point $\hat{P}=(\hat{\kappa}, \hat{\mu}, \hat{\delta})$ close to the curve $C_{1}$, and determine its eigensolutions sensitivities in the direction $\vec{s}$.

A specific numerical case was considered. Let $\hat{P}=(0 \cdot 851,1 \cdot 271,0 \cdot 10)$, the eigenvalues of which are $\hat{\lambda}_{1,3}=-0.079 \pm 0.882 \mathrm{i}$ and $\hat{\lambda}_{2,4}=-0.099 \pm 0.919 \mathrm{i}$; the mistuning is therefore of the order of $4 \%$. The direction $\vec{s}=\{0 \cdot 556,-0 \cdot 831,0\}$ was chosen, along which $\kappa=\hat{\kappa}(1+\eta), \mu=\hat{\mu}(1-\eta)$ and $\delta=\hat{\delta}$.

To apply the modified perturbation method, point $\hat{P}$ could be considered as a perturbation of any point of $C_{1}$ close to $\hat{P}$ : e.g., $P_{1}=(0 \cdot 818,1 \cdot 222,0 \cdot 10)$ (see Figure 7 ), the double eigenvalue of which is $\lambda=-0.091 \pm 0.900 \mathrm{i}$. However, one can ignore the existence of the curve $C_{1}$ and search for a defective point $P_{0}$ in a two-dimensional subspace of the five-dimensional extended space $\Pi^{+}=\left\{\kappa, \mu, \delta, \xi_{1}, \xi_{2}\right\}$; i.e., by fixing $(\kappa, \mu, \delta)=(\hat{\kappa}, \hat{\mu}, \hat{\delta})$ and varying $\xi_{1}$ and $\xi_{2}$. By applying the algorithm of Section 3, $\xi_{1}=\bar{\xi}_{2}=-(2 \cdot 415+3 \cdot 724 \mathrm{i}) 10^{-4}$ is found, together with $A_{0}$ and $\lambda_{0}$. The relevant second order MPE's (equations (24)) are then compared with the second order SPE's (equations $(12 \mathrm{~b}, \mathrm{c}))$ and the exact (numerical) solutions.

In Figure 8 are shown $\operatorname{Re}\left(\tilde{\lambda}_{1,2}\right)$ and $\operatorname{Im}\left(\tilde{\lambda}_{1,2}\right)$ versus the perturbation parameter $\eta$. It is apparent that the MPE's are in good agreement with the exact solution for $\eta \in(-0 \cdot 15,0 \cdot 15)$ (i.e., for modifications of the parameters up to $15 \%)$, whereas the SPS's furnish very poor approximations, although the mistuning is not too small.

An alternative representation of the same results can be obtained by plotting the eigenvalues paths $\tilde{\lambda}_{i}(\eta)$ in the complex plane (see Figure 9). Here a larger interval of $\eta$ is considered; marked points on the curves refer to $\eta=-0 \cdot 15,0,0 \cdot 15,0 \cdot 30$ and $0 \cdot 45$, respectively, and the arrows indicate the directions of the increasing $\eta$ 's. When $\eta=0 \cdot 15$ the two eigenvalues are well separated; when $\eta$ increases they first approach each other roughly on a straight line; then, when $\eta \simeq 0$, they veer abruptly and describe a circle in opposite directions; successively, when $\eta \simeq 0 \cdot 4$, they veer again to come back on to the previous straight line. It is worth noting that the two veerings occur when the modified system $\widetilde{P}$ is close to the defective systems $P_{1}$ and $P_{2}$ (see Figure 7). While the accuracy of the MPE is excellent when $\eta<0$, due to the presence of the second veering, it is less good when $\eta>0$; however, compared with the SPE, the solution is markedly improved.

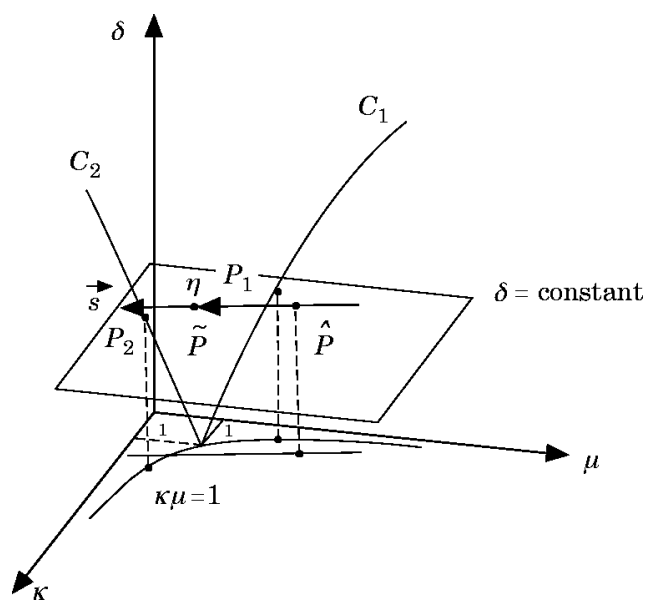

Figure 7. The parameters space for the system (37). 

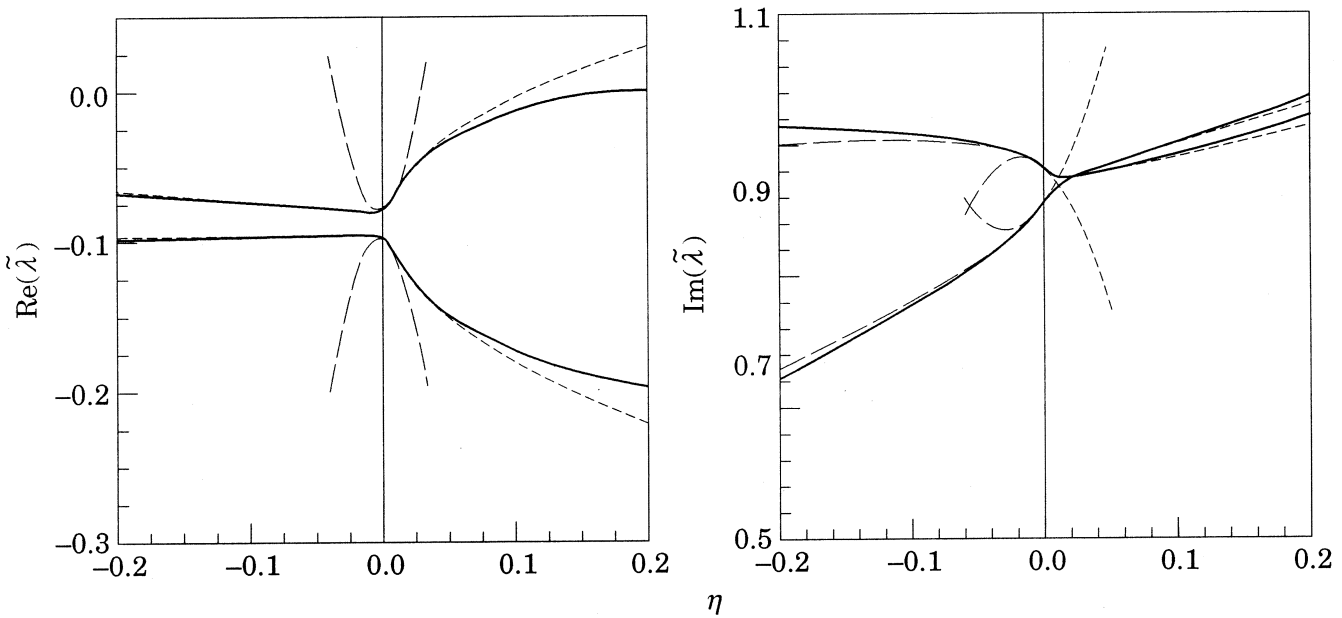

Figure 8. Eigenvalues versus modifications for a two-d.o.f. system. —-, Exact; ----, MPE; ——, SPE.

The eigenvectors $\tilde{u}_{1}(\eta)$ and $\tilde{u}_{2}(\eta)$ are represented in Figure 10. Vectors are normalized according to $e_{4}^{\mathrm{T}} \tilde{u}_{i}=1$ : i.e., by assuming that the displacement of the second oscillator is equal to one. The paths of the other three components are plotted in the complex plane for the same $\eta$ 's considered above. It is found that the second component (the velocity of the second oscillator) varies on a much slower scale than that of the first and third components (velocity and displacement of the first oscillator, respectively), although it exhibits a similar behaviour; in addition, it remains about $\pi / 2$ out of phase with respect to the associated displacement. On the contrary, the velocity and the displacement of the first oscillator vary rapidly, their phase difference being nearly constant, equal to about $\pi / 2$. The paths are straight lines when $\eta$ is less than zero or larger than (about) $0 \cdot 4$, and are curves closely running around the unit

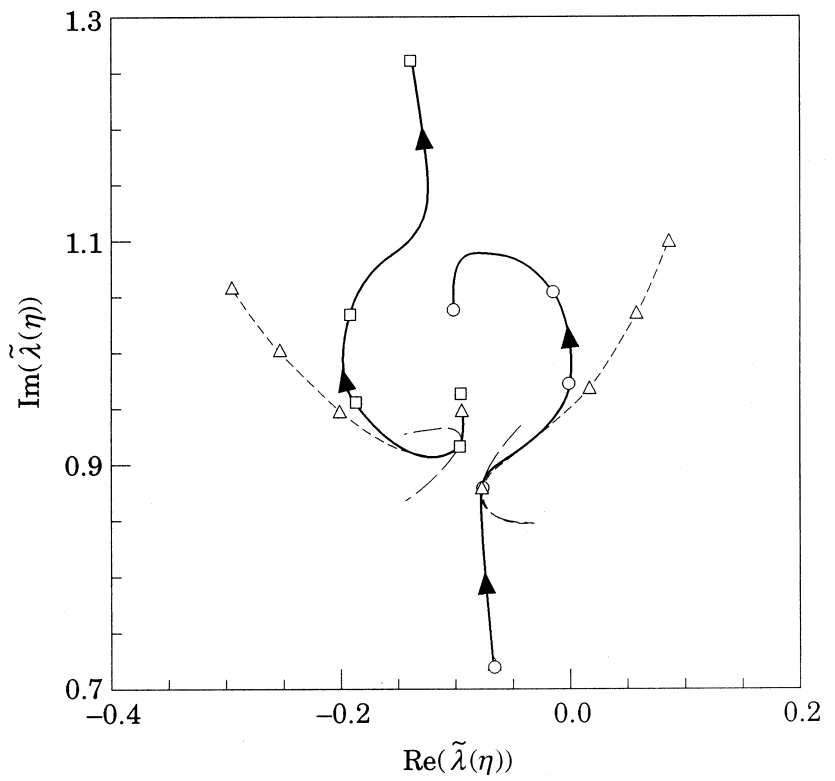

Figure 9. Eigenvalue paths in the complex plane. $\square-\square-\square-\square$, Exact $\tilde{\lambda}_{1} ; \bigcirc-\bigcirc-\bigcirc-\bigcirc$, exact $\tilde{\lambda}_{2} ; \triangle-\triangle-\triangle-\triangle$, MPE; -- , SPE. $\eta_{\min }=-0 \cdot 15 ; \eta_{\max }=0 \cdot 45$. 


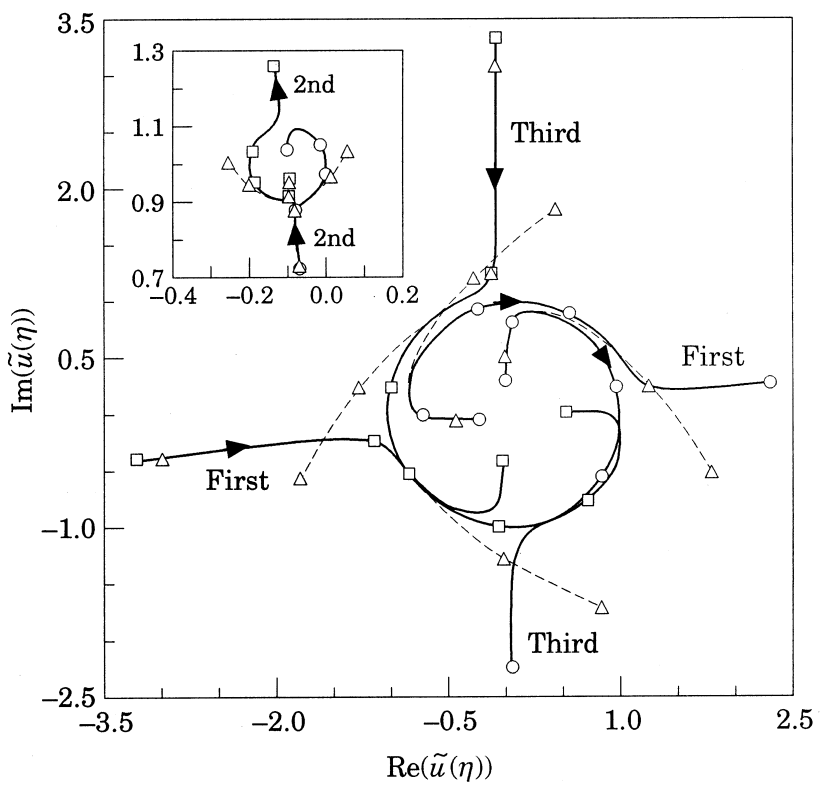

Figure 10. Eigenvector components paths in the complex plane (fourth component equal to one). Exact $\tilde{u}_{1} ; \bigcirc-\bigcirc-\bigcirc-\bigcirc$, exact $\tilde{u}_{2} ; \triangle-\triangle-\triangle-\triangle$, MPE. $\eta_{\min }=-0 \cdot 15 ; \eta_{\max }=0 \cdot 45$.

circle when $\eta$ is in the range $(0,0 \cdot 4)$; the components of the two eigenvectors go along the circle in opposite directions. When $\eta=0 \cdot 198$, i.e., when the two oscillators are equal $(\tilde{\mu}=\tilde{\kappa}=1)$, the two displacements are in phase $(\operatorname{Re}(\tilde{\lambda})=0)$ or in phase opposition $(\operatorname{Re}(\tilde{\lambda})=-0 \cdot 2)$ and are of the same magnitude; when $\eta$ is negative or larger than $0 \cdot 4$ the two displacements are $\pi / 2$ out of phase (forward or backward, respectively), but the magnitudes are quite different, since in each mode one component prevails. As for the eigenvalues, the MPE's furnish good approximations of the exact solution for moderate $\eta$; SPE's, not shown, again give poor approximations.

\section{REMARKS AND CONCLUSIONS}

Three remarks may be made on the method proposed.

(1) The problem treated here has some similarity with localization and loci veering phenomena, already studied in depth (e.g., see the paper by Pierre [15] and reference [13] for helpful comments). In those problems too, the eigenvalues depend on two small parameters: an imperfection (modification) parameter $\eta$ and a coupling parameter (mistuning) $\Delta$. Pierre also utilized perturbation methods: when the $\eta / \Delta$ ratio was small he assumed $\eta$ as the perturbation parameter; when the ratio was large, he considered $\Delta$ as the perturbation parameter and included $\eta$ in the unperturbed system to split its eigenvalues. In all cases, series of integer powers were used, since only non-defective (conservative) systems were taken into account. Pierre's method could also be applied to the problem considered here; however, it would require the solution of a new eigenvalue problem, after having solved the unmodified problem, in contrast with the main scope of the sensitivity analysis. Therefore, in the method proposed, $\Delta$ and $\eta$ are considered together to be perturbations of an ideal system with repeated eigenvalues. Since the unperturbed system is defective, fractional powers are used.

(2) The key point of the algorithm is to build the tuned system $A_{0}$, after the unmodified system $\hat{A}$ has been given. The basic idea of Section 3 is to perturb the canonical Jordan form 
of $A_{0}$ by introducing suitable new parameters $\xi_{i}$, and equating the perturbed eigenvalues to the unmodified ones. In this way the parameters $\xi_{i}$ and $\lambda_{0}$ can be found. Therefore, if only two eigenvalues $\hat{\lambda}_{k}$ are nearly coincident, the unique parameter $\xi_{1}$, together with $\lambda_{0}$, is sufficient for the purpose; if three eigenvalues $\hat{\lambda}_{k}$ are nearly coincident, two parameters $\xi_{1}$ and $\xi_{2}$ must be introduced to perturb the Jordan block, and so on. Therefore the method can easily be extended to more complex cases of several nearly coincident eigenvalues. Some details on the topic are given in the Appendix.

(3) The idea of extending the parameter space in order to find a coalescence point close to the actual point already underlies reference [13]. There, in fact, the perturbation parameter $\eta$ was allowed to assume complex values and therefore its imaginary part had a rôle equivalent to that of the parameter $\xi_{1}$ of the present method. However, only the direct problem was dealt with in reference [13], and the theory cannot be extended in a simple way to solve the inverse problem, if the general case of several nearly coincident eigenvalues is considered.

In closing, the following conclusions can be drawn.

(1) A linear system is nearly defective if two or more eigenvalues are nearly coincident and the associated eigenvectors nearly parallel. It can be considered as a perturbation of an ideal, tuned, defective system having perfectly coincident eigenvalues and eigenvectors.

(2) If modified eigensolutions are evaluated by extrapolating the unmodified nearly coincident eigensolutions, SPE's are obtained in terms of integer powers of the modification parameter $\eta$. The SPE's are uniformly valid only in a very small interval of $\eta$, so they are not useful for practical purposes.

(3) If modified eigensolutions are extrapolated by the tuned system, MPE's are obtained in terms of fractional powers of two parameters, the mistuning and the modification parameters. MPE's are uniformly valid for any $\eta$, except for critical modifications having the character of singular perturbations.

(4) A geometric interpretation of the singular perturbations has been given: they occur when the modification is tangent to the locus of the defective systems in the space of the parameters.

(5) To apply MPE's a tuned system close to the mistuned system should be known. Since in general this is not the case, an ideal tuned system can be artfully built by suitably extending the space of the parameters. The tuned system obtained does not represent any physical system, being only a mathematical tool to solve the problem.

(6) The efficiency of the method has been tested on simple examples. In particular, good accord between exact and perturbational solutions has been found up to modifications of $15 \%$, although an unfavorable case has been considered.

\section{ACKNOWLEDGMENTS}

This research has been supported by the Italian Ministry of the University, Scientific and Technological Research (MURST 40\% funds).

\section{REFERENCES}

1. J. H. Wilkinson 1965 The Algebraic Eigenvalue Problem. Oxford: Clarendon Press.

2. T. Kato 1982 A Short Introduction to Perturbation Theory for Linear Operators. New York: Springer-Verlag.

3. H. M. Adelman and R. T. HaftKa 1986 American Institute of Aeronautics and Astronautics Journal 24(5), 823-832. Sensitivity analysis of discrete systems.

4. D. V. MURTHY and R. T. HAFTKA 1988 International Journal for Numerical Methods in Engineering 26, 293-311. Derivatives of eigenvalues and eigenvectors of a general complex matrix. 
5. J. A. BRANDON 1990 Strategies for Structural Dynamic Modification. New York: John Wiley.

6. W. C. Mills-Curran 1988 American Institute of Aeronautics and Astronautics Journal 26(7), 867-871. Calculation of eigenvectors derivatives for structures with repeated eigenvalues.

7. R. L. DaILEy 1989 American Institute of Aeronautics and Astronautics Journal 27(4), 486-491. Eigenvector derivatives with repeated eigenvalues.

8. G. J. How and S. P. KenNy 1992 American Institute of Aeronautics and Astronautics Journal 30(9), 2317-2324. Eigenvalue and eigenvector approximate analysis for repeated eigenvalue problems.

9. J. Juang, P. Ghaemmaghami and K. B. Lim 1989 Journal of Guidance, Control and Dynamics 12(4), 480-486. Eigenvalue and eigenvector derivatives of a nondefective matrix.

10. B. Friedman 1956 Principles and Techniques of Applied Mathematics. New York: John Wiley.

11. A. Luongo 1994 American Institute of Aeronautics and Astronautics Journal 31(7), 1321-1328. Eigensolutions sensitivity for nonsymmetric matrices with repeated eigenvalues.

12. A. LuONGo 1995 American Institute of Aeronautics and Astronautics Journal 33(1), 120-127. Free vibrations and sensitivity analysis of a defective two-degree-of-freedom system.

13. M. S. Triantafyllou and G. S. Triantafyllou 1991 Journal of Sound and Vibration 150, 485-500. Frequency coalescence and mode localization phenomena: a geometric theory.

14. W. T. KoITER 1945 Doctoral Thesis, University of Delft. On the stability of elastic equilibrium. (English translation NASA Techn. Trans. F-10, 833, 1967).

15. C. PIERre 1988 Journal of Sound and Vibration 126, 485-502. Mode localization and eigenvalue loci veering phenomena in disordered structures.

\section{APPENDIX: THE INVERSE PROBLEM IN THE GENERAL CASE}

An outline of how to extend the algorithm of Section 3 to the more general case of several nearly coincident eigenvalues with associated nearly parallel eigenvectors is given here.

Let $\hat{\lambda}_{1}, \hat{\lambda}_{2}, \ldots, \hat{\lambda}_{\rho}$ be $\rho$ nearly-coincident eigenvalues of $\hat{A}$, the corresponding right and left nearly parallel eigenvectors of which are collected in the $N \times \rho$ matrices $\hat{U}_{\rho}$ and $\hat{V}_{\rho}$, respectively. The unknown ideal defective matrix $A_{0}$ has an eigenvalue $\lambda_{0}$ of multiplicity $m=\rho$ and associated generalized eigenvectors matrices $X_{\rho}$ and $Y_{\rho}$, of dimensions $N \times \rho$. The $\rho \times \rho$ Jordan block $J_{\rho}$ of $A_{0}$ is perturbed by $\rho-1$ parameters $\xi_{j}$ as follows:

$$
\hat{J}_{\rho}=J_{\rho}+\delta J_{\rho}=\left[\begin{array}{cccccc}
\lambda_{0} & 1 & & & \\
& \lambda_{0} & 1 & & & \\
& & \ddots & & \ddots & \\
& & \lambda_{0} & & 1 \\
& & & & \\
\xi_{1} & \xi_{2} \cdots & \xi_{\rho-1} & \lambda_{0}
\end{array}\right] .
$$

Consequently, the perturbation matrix $\delta A=X_{\rho} \delta J_{\rho} Y_{\rho}^{\mathrm{H}}$ is

$$
\delta A=\xi_{1} x_{\rho} y_{1}^{\mathrm{H}}+\xi_{2} x_{\rho} y_{2}^{\mathrm{H}}+\cdots+\xi_{\rho-1} x_{\rho} y_{\rho-1}^{\mathrm{H}} .
$$

The parameters $\xi_{j}$ and $\lambda_{0}$ must be chosen in such a way that $\hat{J}_{\rho}$ has $\rho$ eigenvalues equal to $\hat{\lambda}_{1}, \hat{\lambda}_{2}, \ldots, \hat{\lambda}_{\rho}$ : i.e.,

$$
\operatorname{det}\left(\hat{J}_{\rho}-\lambda I\right)=\prod_{k=1}^{\rho}\left(\lambda-\hat{\lambda}_{k}\right) \quad \forall \lambda
$$

From the previous identity $\rho$ algebraic equations are obtained, which can be solved in sequence for the unknowns $\lambda_{0}, \xi_{\rho-1}, \xi_{\rho-2}, \ldots, \xi_{1}$. In particular, $\lambda_{0}=\left(\sum_{k=1}^{\rho} \hat{\lambda}_{k}\right) / \rho$ is found: i.e., $\lambda_{0}$ is the centre of mass of the points $\hat{\lambda}_{k}$ on the complex plane.

$\hat{J}_{\rho}$ admits the spectral representation $\hat{J}_{\rho}=R_{\rho} \hat{\Lambda}_{\rho} S_{\rho}^{\mathrm{H}}$, where $\hat{\Lambda}_{\rho}=\operatorname{diag}\left(\hat{\lambda}_{1}, \hat{\lambda}_{2}, \ldots, \hat{\lambda}_{\rho}\right)$ and $R_{\rho}$ and $S_{\rho}=R_{\rho}^{-\mathrm{H}}$ are $\rho \times \rho$ matrices collecting its right and left eigenvectors, respectively. Therefore, the perturbed matrix $A_{0}+\delta A$ has proper eigenvectors matrices $\hat{U}_{0 \rho}=X_{\rho} R_{\rho}$ and 
$\hat{V}_{0 \rho}=Y_{\rho} S_{\rho}$. By requiring $\hat{U}_{0 \rho}=\hat{U}_{\rho}$ and $\hat{V}_{0 \rho}=\hat{V}_{\rho}$ and solving with respect to the unknown quantities, one finds that

$$
X_{\rho}=\hat{U}_{\rho} R_{\rho}^{-1}, \quad Y_{\rho}=\hat{V}_{\rho} R_{\rho}^{\mathrm{H}} .
$$

From equations (A4) the generalized eigenvectors $x_{1}, x_{2}, \ldots, x_{\rho}$ and $y_{1}, y_{2}, \ldots, y_{\rho}$ are obtained. However, they generally do not satisfy the requested normalization conditions $e_{h}^{\mathrm{T}} x_{1}=1, e_{h}^{\mathrm{T}} x_{j}=0(j>1)$ and $y_{i}^{\mathrm{H}} x_{j}=\delta_{i j}$. Therefore a new set of normalized eigenvectors $x_{k}^{*}=x_{k}+a_{k} x_{1}$ and $y_{k}^{*}=y_{k}+b_{k} y_{\rho}$ must be constructed. By using the new vectors, $\delta A$ can be evaluated from equation (A2) and $A_{0}=\hat{A}-\delta A$ computed as a difference. Obviously, if more groups of nearly coincident eigenvalues occurs, the relevant perturbation matrices $\delta A_{i}$ must be added among them, as already seen in Section 3.

Finally, it should be noted that, when equations (18) and (19) are used (with $\delta A$ instead of $\left.\xi A_{\xi}\right), \delta_{1} \lambda_{k}$ is found to depend on $\xi_{1}$ only, $\delta_{2} \lambda_{k}$ on $\xi_{1}$ and $\xi_{2}$ and so on; moreover, $u_{*}$ is independent of the $\xi$ 's, as in the particular case $\rho=2$. 